CONFORMAL GEOMETRY AND DYNAMICS

An Electronic Journal of the American Mathematical Society

Volume 3, Pages 102-115 (August 16, 1999)

S $1088-4173(99) 00026-0$

\title{
RESTRICTIONS ON HARMONIC MORPHISMS
}

\author{
M. T. MUSTAFA
}

\begin{abstract}
We consider horizontally (weakly) conformal maps $\phi$ between Riemannian manifolds and calculate a formula for the Laplacian of the dilation of $\phi$, using the language of moving frames. Applying this formula to harmonic horizontally (weakly) conformal maps or equivalently to harmonic morphisms we obtain a Weitzenböck formula similar to an earlier result of the author ( $\mathrm{J}$. London Math. Soc. (2) 57 (1998), 746-756), and hence vanishing results for harmonic morphisms from compact manifolds of positive curvature. Further, a method is developed to obtain restrictions on harmonic morphisms from some non-compact and non-positively curved domains. Finally, a discussion of restrictions on harmonic morphisms between simply connected space forms is given.
\end{abstract}

\section{INTRODUCTION}

The maps under consideration are harmonic morphisms between Riemannian manifolds, i.e. the maps which preserve germs of harmonic functions. Such maps are characterized as a subclass of harmonic maps (see below). In [17], the author presented a Bochner technique for harmonic morphisms by extending the Bochner type formula for harmonic maps [6] and gave its consequences for harmonic morphisms from compact domain manifolds.

This note has a twofold purpose. First is to represent the Bochner type technique for harmonic morphisms in the language of frames. Secondly, to improve the results of [17], by obtaining applications of the Bochner type formula, for harmonic morphisms from a larger class of domain manifolds. Thus along with non-existence results of [17], we get restrictions on harmonic morphisms from compact as well as non-compact manifolds. This allows us to get a clearer picture of the theory of harmonic morphisms between simply-connected space forms. For example, we show in the final section

1. There exist no non-constant harmonic morphisms $\phi: \mathbb{R}^{m} \rightarrow \mathbb{H}^{n}$.

2. Let $\phi: M^{m} \rightarrow N^{n}$ be a harmonic morphism with the dilation $\lambda$. If $\left(M^{m}, N^{n}\right)=$ $\left(\mathbb{H}^{m}, \mathbb{H}^{n}\right)$, then $\lambda^{2} \leq((m-1) /(n-1))$. On the other hand if $\phi$ is submersive and $\left(M^{m}, N^{n}\right)=\left(\mathbb{S}^{m}, \mathbb{S}^{n}\right)$, then $\lambda^{2}>((m-1) /(n-1))$.

After giving the necessary framework in Sections 2, 3 we compute the Laplacian in Section 4. Section 5 consists of the results on restrictions on harmonic morphisms between general Riemannian manifolds while the restrictions on harmonic morphisms between simply-connected space forms are discussed in Section 6 .

Received by the editors December 29, 1997 and, in revised form, June 8, 1999.

1991 Mathematics Subject Classification. Primary 58E20, 53C20.

Key words and phrases. Harmonic morphisms, harmonic maps, Bochner technique.

(C)1999 American Mathematical Society 


\section{NOtATION AND PRELIMINARIES}

In this section, after fixing the notation we explain some fundamental relations which will be used repeatedly in the subsequent sections.

Let $\phi:\left(M^{m}, g\right) \rightarrow\left(N^{n}, h\right)$ be a smooth mapping from an $m$-dimensional Riemannian manifold with the metric $g$ to an $n$-dimensional Riemannian manifold with the metric $h$ and denote the pull back of the tangent bundle on $M$ by $E=\phi^{-1} T N$. Throughout this article we assume the following conventions, unless otherwise stated.

The indices range as: $\quad 1 \leq a, b, c \leq m ; \quad 1 \leq i, j, k \leq n ; \quad n+1 \leq \alpha, \beta, \gamma$.

The operators on the bundles $T M, T N, E$ will be denoted respectively by the superscripts $M, N, \phi$, e.g. the respective connections will be denoted as $\nabla^{M}, \nabla^{N}$, $\nabla^{\phi}$.

With the above setting, the following relations are well known.

2.1. Let $\left\{X_{a}\right\}_{a=1}^{m},\left\{Y_{i}\right\}_{i=1}^{n}$ be local orthonormal framings on $M, N$ respectively and $\left\{e_{i}\right\}_{i=1}^{n}$ be the induced framing on $E$ defined by $e_{i}=Y_{i} \circ \phi$, then there exist smooth local coframings $\left\{\omega_{a}\right\}_{a=1}^{m},\left\{\eta_{i}\right\}_{i=1}^{n}$ and $\left\{\phi^{*} \eta_{i}\right\}_{i=1}^{n}$ on $T M, T N$ and $E$ respectively such that (locally)

$$
g=\sum_{a=1}^{m} \omega_{a}^{2} \quad \text { and } \quad h=\sum_{i=1}^{n} \eta_{i}^{2} .
$$

The corresponding first structure equations are:

$$
\begin{array}{ll}
d \omega_{a}=\sum_{b=1}^{m} \omega_{b} \wedge \omega_{b a}, & \omega_{a b}=-\omega_{b a}, \\
d \eta_{i}=\sum_{j=1}^{n} \eta_{j} \wedge \eta_{j i}, & \eta_{i j}=-\eta_{j i}, \\
d\left(\phi^{*} \eta_{i}\right)=\sum_{j=1}^{n} \phi^{*} \eta_{j} \wedge \phi^{*} \eta_{j i}, & \phi^{*} \eta_{i j}=-\phi^{*} \eta_{j i},
\end{array}
$$

where the unique 1 -forms $\omega_{a b}, \eta_{i j}, \phi^{*} \eta_{i j}$ are the respective connection forms. The second structure equations are as follows.

$$
\begin{aligned}
& d \omega_{a b}=\sum_{c=1}^{m} \omega_{a c} \wedge \omega_{c b}+\Omega_{a b}^{M} ; \quad d \eta_{i j}=\sum_{k=1}^{n} \eta_{i k} \wedge \eta_{k j}+\Omega_{i j}^{N} ; \\
& d\left(\phi^{*} \eta_{i j}\right)=\sum_{k=1}^{n} \phi^{*} \eta_{i k} \wedge \phi^{*} \eta_{k j}+\phi^{*} \Omega_{i j}^{N}
\end{aligned}
$$

where the curvature 2 -forms are given by

$$
\Omega_{a b}^{M}=-\frac{1}{2} \sum_{c, d=1}^{m} \mathbf{R}_{a b c d}^{M} \omega_{c} \wedge \omega_{d} \quad \text { and } \quad \Omega_{i j}^{N}=-\frac{1}{2} \sum_{k, l=1}^{n} \mathbf{R}_{i j k l}^{N} \eta_{k} \wedge \eta_{l} .
$$

2.2. The pull back map $\phi^{*}$ and the push forward map $\phi_{*}$ can be written as

$$
\phi^{*} \eta_{i}=\sum_{a=1}^{m} f_{i a} \omega_{a} \quad \text { for unique functions } f_{i a} \text { on } U \subset M \text {. }
$$




$$
\phi_{*}=\sum_{i=1}^{n} e_{i} \otimes \phi^{*} \eta_{i}=\sum_{i=1}^{n} \sum_{a=1}^{m} f_{i a} e_{i} \otimes \omega_{a}
$$

Note that $\phi_{*}$ is a section of the vector bundle $\phi^{-1} T N \otimes T^{*} M$.

2.3. The covariant differential operators are represented as

$$
\nabla^{M} X_{a}=\sum_{b=1}^{m} \omega_{a b} \otimes X_{b}, \quad \nabla^{N} Y_{i}=\sum_{j=1}^{n} \eta_{i j} \otimes Y_{j},
$$

and $\quad \nabla^{*} \omega_{a}=-\sum_{c=1}^{m} \omega_{c a} \otimes \omega_{c}, \quad$ where $\nabla^{*}$ is the connection on $T^{*} M$.

Furthermore, the induced connection $\nabla^{\phi}$ on $E$ is

$$
\nabla^{\phi} e_{i}=\sum_{j, k=1}^{n} \sum_{a=1}^{m}\left(\eta_{i j}\left(Y_{k}\right) \circ \phi\right) e_{j} \otimes f_{k a} \omega_{a} .
$$

This can be checked by using $\S 2.2$ and above in the definition of the pull back connection i.e. $\nabla^{\phi} e_{i}(X)=\nabla^{\phi} e_{i}=\left(\nabla_{\phi_{*} X}^{N} Y_{i}\right) \circ \phi$ for $X \in \mathcal{C}(T M)$.

2.4. The components of the Ricci tensor and scalar curvature are defined respectively by

$$
\mathbf{R}_{a b}^{M}=\sum_{c=1}^{m} \mathbf{R}_{a c b c}^{M} \quad \text { and } \quad \mathbf{S c a l}^{M}=\sum_{a=1}^{m} \mathbf{R}_{a a}^{M}
$$

2.5. Given a function $f: M \rightarrow \mathbb{R}$, there exist unique functions $f_{c b}=f_{b c}$ such that

$$
d f_{c}-\sum_{b=1}^{m} f_{b} \omega_{c b}=\sum_{b=1}^{m} f_{c b} \omega_{b}
$$

where $f_{c}=d f\left(X_{c}\right)$ for a local orthonormal frame $\left\{X_{c}\right\}_{c=1}^{m}$.

Proof. Taking the exterior derivative of $d f=\sum_{c=1}^{m} f_{c} \omega_{c}$ and using structure equations, we have

$$
\begin{aligned}
0 & =\sum_{c=1}^{m}\left[d f_{c} \wedge \omega_{c}+\sum_{b=1}^{m} f_{b c} \omega_{b} \wedge \omega_{b c}\right] \\
& =\sum_{c=1}^{m}\left[\left(d f_{c}-\sum_{b=1}^{m} f_{b} \omega_{c b}\right) \wedge \omega_{c}\right] .
\end{aligned}
$$

Hence by Cartan's lemma (cf. [20]), there exist unique functions $f_{c b}=f_{b c}$ such that

$$
d f_{c}-\sum_{b=1}^{m} f_{b} \omega_{c b}=\sum_{b=1}^{m} f_{c b} \omega_{b}
$$


2.6. The Laplacian of a function $f$ on $M$ is given by

$$
\Delta f=-\operatorname{trace} \nabla d f
$$

that is, the negative of the usual Laplacian on functions.

Lemma 2.1. The Laplacian of a function $f: M \rightarrow \mathbb{R}$ satisfies

$$
-\Delta f=\sum_{c=1}^{m} \nabla d f\left(X_{c}, X_{c}\right)=\sum_{c=1}^{m} f_{c c}
$$

where the functions $f_{c c}$ are defined by Equation 2.1.

Proof. First notice that

$$
\begin{aligned}
\nabla d f\left(X_{d}, X_{c}\right) & =\left(\nabla_{X_{d}} d f\right) X_{c}=X_{d}\left(d f\left(X_{c}\right)\right)-d f\left(\nabla^{M} X_{d} X_{c}\right) \\
& =\left(d f_{c}-\sum_{b=1}^{m} f_{b} \omega_{c b}\right)\left(X_{d}\right)=\sum_{b=1}^{m} f_{c b} \omega_{b}\left(X_{d}\right) .
\end{aligned}
$$

Hence $\nabla d f\left(X_{c}, X_{c}\right)=f_{c c}$.

\section{SECOND FUNDAMENTAL FORM, HARMONIC MAPS AND HARMONIC MORPHISMS}

Let $\phi: M \rightarrow N$ be a smooth map. As seen above, $\phi_{*}$ is a section of $\phi^{-1} T N \otimes$ $T^{*} M$. If $\tilde{\nabla}$ denotes the connection on the bundle $\phi^{-1} T N \otimes T^{*} M$, then the quadratic form $\tilde{\nabla} \phi_{*}$ is called the second fundamental form of $\phi$.

Lemma 3.1. Keeping the notation of §2.2, there exist unique symmetric functions $f_{i a b}=f_{i b a}$ on $U \subset M$ such that (locally) the second fundamental form of a map $\phi$ is given by

$$
\tilde{\nabla} \phi_{*}=\sum_{i=1}^{n} \sum_{a, b=1}^{m} f_{i a b} e_{i} \otimes \omega_{b} \otimes \omega_{a}
$$

where the components $f_{i a b}$ satisfy

$$
\sum_{b=1}^{m} f_{i a b} \omega_{b}=d f_{i a}+\sum_{j=1}^{n} f_{j a} \phi^{*} \eta_{j i}+\sum_{b=1}^{m} f_{i b} \omega_{b a} .
$$

Proof. Differentiating

$$
\phi_{*}=\sum_{i=1}^{n} \sum_{a=1}^{m} f_{i a} e_{i} \otimes \omega_{a}
$$

we have

$$
\begin{gathered}
\tilde{\nabla} \phi_{*}=\sum_{i=1}^{n} \sum_{a=1}^{m}\left[\nabla^{\phi} e_{i} \otimes f_{i a} \omega_{a}+e_{i} \otimes d f_{i a} \otimes \omega_{a}+f_{i a} e_{i} \otimes \nabla^{* M} \omega_{a}\right] \\
=\sum_{i, a}\left[f_{i a} \sum_{j, k=1}^{n} \sum_{b=1}^{m}\left\{\left(\eta_{i j}\left(Y_{k}\right) \circ \phi\right) e_{j} \otimes f_{k b \omega_{b}}\right\}\right. \\
\left.\otimes \omega_{a}+e_{i} \otimes d f_{i a} \otimes \omega_{a}+f_{i a} e_{i} \otimes \sum_{b=1}^{m}\left(\omega_{a b} \otimes \omega_{b}\right)\right] .
\end{gathered}
$$


Noticing that

$$
\sum_{i, j, k=1}^{n} \sum_{a, b=1}^{m} f_{i a} f_{k b}\left(\eta_{i j}\left(y_{k}\right) \circ \phi\right) e_{j}=\sum_{i, j, k=1}^{n} \sum_{a, b=1}^{m} f_{j a} f_{k b}\left(\eta_{j i}\left(y_{k}\right) \circ \phi\right) e_{i}
$$

and using

$$
\phi^{*} \eta_{j i}=\sum_{b=1}^{m} \phi^{*} \eta_{j i}\left(X_{b}\right) \omega_{b}=\sum_{b=1}^{m} \sum_{k=1}^{n} f_{k b}\left(\eta_{j i}\left(Y_{k}\right) \circ \phi\right) \omega_{b}
$$

we get the required relation. The uniqueness and symmetry in $a, b$ of the functions $f_{i a b}$ follows by applying Cartan's lemma (cf. [16, p. 3]).

Definition 3.2. A smooth map $\phi: M \rightarrow N$ is called totally geodesic if its second fundamental form vanishes i.e. if $f_{i a b}=0$.

The tension field $\tau(\phi)$ of a smooth map $\phi$, as defined by Eells-Sampson in [6], is the vector field given by trace $\tilde{\nabla} \phi_{*}$.

Definition 3.3 ([6]). A smooth map $\phi: M \rightarrow N$ is said to be harmonic if and only if it extremizes the associated energy integral $E(\phi)=\frac{1}{2} \int_{\Omega}\left\|\phi_{*}\right\|^{2} d v^{M}$ for every compact domain $\Omega \subset M$.

It is well-known, cf. $[6,4,5]$, that a map $\phi$ is harmonic if and only if its tension field is zero, i.e. if and only if $\sum_{a} f_{i a a}=0$.

The notions of horizontally conformal maps and harmonic morphisms were formally introduced independently by B. Fuglede [8] and T. Ishihara [13]. In a sense, the former can be thought of as a generalization of the concept of Riemannian submersions and the latter can be thought of as a special class of harmonic maps. Here we present the basic definitions, and refer to $[8,1,21]$ for the fundamental results and properties. An account of the theory of harmonic morphisms in the language of moving frames is given in [3]. An updated bibliography of harmonic morphisms can be found on the INTERNET by linking to [12].

For a smooth map $\phi: M^{m} \rightarrow N^{n}$, let $C_{\phi}=\left\{x \in M \mid \operatorname{rank} \phi_{* x}<n\right\}$ and let $M^{*}$ denote the set $M \backslash C_{\phi}$.

Definition 3.4. Let $\phi:\left(M^{m}, g\right) \rightarrow\left(N^{n}, h\right)$ be a smooth map. Choose $U \subset M$, $V \subset N$ with $U \subset \phi^{-1}(V)$ and set $\phi^{*}\left(\eta_{i}\right)=\sum_{a} f_{i a} \omega_{a}$, (notation as in $\S 2$ ). Then $\phi$ is called horizontally (weakly) conformal if there exists a function $\lambda \geq 0$ on $U$ such that $\lambda>0$ on $U \cap M^{*}$ with

$$
f_{i a} f_{j a}=\lambda^{2} \delta_{i j}, \quad 1 \leq a \leq m, \quad 1 \leq i, j \leq n
$$

and $\lambda=0$ on $U \cap C_{\phi}$.

The function $\lambda$ is termed as the dilation of the horizontally (weakly) conformal map. This definition is based on $[3, \S 1]$, see [8] for an alternative description.

\section{Remark 3.5.}

1. The dilation $\lambda$ satisfies $n \lambda^{2}=\left\|\phi_{*}\right\|^{2}$.

2. $\phi: M^{*} \rightarrow N^{n}$ is submersive, i.e. $\phi_{*}$ has rank $n$ everywhere on $M^{*}$.

3. Given a horizontally (weakly) conformal map $\phi:\left(M^{m}, g\right) \rightarrow\left(N^{n}, h\right)$ with dilation $\lambda$. For each $x \in M^{*}$, we can choose an orthonormal coframing $\left\{\omega_{a}\right\}_{a=1}^{m}$ 
such that (locally)

$$
g=\sum_{i=1}^{n} \omega_{i}^{2}+\sum_{\alpha=n+1}^{m} \omega_{\alpha}^{2}
$$

where $\left\{\omega_{i}\right\}_{i=1}^{n}$ is a local orthonormal coframing of the subbundle $\left(\operatorname{Ker} \phi_{*}\right)^{\perp}$ which satisfies $\lambda \omega_{i}=\phi^{*} \eta_{i}$ and $\left\{\omega_{\alpha}\right\}_{\alpha=n+1}^{m}$ is a local orthonormal coframing of $\operatorname{Ker} \phi_{*}$. We call the subbundles $\left(\operatorname{Ker} \phi_{*}\right)^{\perp}$ and $\operatorname{Ker} \phi_{*}$ as the horizontal and vertical subbundles. Such a coframing is named, by R. L. Bryant [3], as $\phi$-adapted.

Hence, we see that for a horizontally (weakly) conformal map the functions $f_{i a}$ defined by $\phi^{*} \eta_{i}=\sum_{a} f_{i a} \omega_{a}$ satisfy $f_{i a}=\lambda \delta_{i a}$ with respect to a $\phi$-adapted coframing. This observation will be important in the next section.

Harmonic morphisms are maps which preserve Laplace's equation in the following sense.

Definition 3.6. A smooth map $\phi: M^{m} \rightarrow N^{n}$ is called a harmonic morphism if, for every real-valued function $f$ which is harmonic on an open subset $V$ of $N$ with $\phi^{-1}(V)$ non-empty, $f \circ \phi$ is a real-valued harmonic function on $\phi^{-1}(V) \subset M$.

These are related to harmonic maps and horizontally (weakly) conformal maps via the following characterization, obtained in $[8,13]$.

A smooth map is a harmonic morphism if and only if it is harmonic and horizontally (weakly) conformal.

In terms of local coframings, it can be stated as (see [3]):

A smooth map $\phi$ is a harmonic morphism if and only if locally it satisfies

$$
f_{i a a}=0 \quad \text { and } \quad f_{i a} f_{j a}=\lambda^{2} \delta_{i j} \quad \text { for some function } \lambda^{2} \geq 0
$$

where $\lambda$ denotes the dilation of $\phi$ and $f_{i a a}, f_{i a}, f_{j a}$ are as in §2.

Although the above characterization says that harmonic morphisms may be viewed as a subclass of harmonic maps, it is important to notice that in certain cases harmonic morphisms have properties which are exactly dual to the properties of harmonic maps (see the explanation by J. C. Wood in [22]).

\section{LAPLACIAN OF $\lambda^{2}$}

Recall that the notation of previous sections will be followed throughout this section.

Consider a horizontally (weakly) conformal map $\phi$ with dilation $\lambda$. We wish to calculate $-\Delta \lambda^{2}$, for which we need the following lemma.

Lemma 4.1. Let $\phi:\left(M^{m}, g\right) \rightarrow\left(N^{n}, h\right)$ be a horizontally (weakly) conformal map with dilation $\lambda$. Then

1.

$$
n d \lambda^{2}=2 \sum_{i=1}^{n} \sum_{a, b=1}^{m} f_{i a} f_{i a b} \omega_{b}
$$

In particular, if $\phi$ is totally geodesic, then $\lambda$ is constant; a well-known result (cf. $[4, \S 3.1])$. 
2. The differential of the functions $f_{i a b}$ satisfies

$$
\begin{aligned}
\sum_{b=1}^{m}\left[d f_{i a b}+\sum_{c=1}^{m} f_{i a c} \omega_{c b}+\sum_{c=1}^{m} f_{i c b} \omega_{c a}+\sum_{j=1}^{n} f_{j a b} \phi^{*} \eta_{j i}\right] \wedge \omega_{b} & \\
& =\sum_{b=1}^{m} f_{i b} \Omega_{b a}^{M}+\sum_{j=1}^{n} f_{j a} \phi^{*} \Omega_{j i}^{N} .
\end{aligned}
$$

If the 1-form on the left hand side of the above equation is written as

$$
d f_{i a b}+\sum_{c=1}^{m} f_{i a c} \omega_{c b}+\sum_{c=1}^{m} f_{i c b} \omega_{c a}+\sum_{j=1}^{n} f_{j a b} \phi^{*} \eta_{j i}=\sum_{c=1}^{m} f_{i a b c} \omega_{c},
$$

then the functions $f_{i a b c}$ satisfy the following curvature identity:

$$
f_{i a b c}-f_{i a c b}=-\sum_{d=1}^{m} f_{i d} \mathbf{R}_{d a b c}^{M}-\sum_{j, k, l=1}^{n} f_{j a} f_{k c} f_{l b} \mathbf{R}_{j i k l}^{N} .
$$

Proof.

1. Differentiating

$$
n \lambda^{2}=\left\|\phi_{*}\right\|^{2}=\sum_{i=1}^{n} \sum_{a=1}^{m}\left(f_{i a}\right)^{2} \quad(\text { see } \S 2.2)
$$

we have

$$
n d \lambda^{2}=\sum_{i=1}^{n} \sum_{a=1}^{m} 2 f_{i a} d f_{i a} .
$$

Using the expression for $d f_{i a}$ from Lemma 3.1, we have

$$
n d \lambda^{2}=2 \sum_{i=1}^{n} \sum_{a=1}^{m}\left[\sum_{b=1}^{m} f_{i a} f_{i a b} \omega_{b}-\sum_{j=1}^{n} f_{i a} f_{j a} \phi^{*} \eta_{j i}-\sum_{b=1}^{m} f_{i a} f_{i b} \omega_{b a}\right] .
$$

The last two terms vanish due to skew symmetry of $\omega_{b a}$ and $\phi^{*} \eta_{j i}$, and we get the first part. The final statement follows from the fact that the functions $f_{i a b}$ are components of the second fundamental form of $\phi$.

2. Differentiation of Equation 3.1 implies

$$
\begin{aligned}
\sum_{b=1}^{m}\left[d f_{i a b}\right. & \left.\wedge \omega_{b}+f_{i a b} d \omega_{b}\right] \\
=0 & +\sum_{j=1}^{n}\left[d f_{j a} \wedge \phi^{*} \eta_{j i}+f_{j a} d \phi^{*} \eta_{j i}\right]+\sum_{b=1}^{m}\left[d f_{i b} \wedge \omega_{b a}+f_{i b} d \omega_{b a}\right] .
\end{aligned}
$$

The following relations can be established easily, by using structure equations and Equation 3.1.

$$
\begin{gathered}
\sum_{b, c=1}^{m} f_{i a c} \omega_{c b} \wedge \omega_{b}=\sum_{c=1}^{m} f_{i a c} d \omega_{c} \\
\sum_{b, c=1}^{m} f_{i c b} \omega_{c a} \wedge \omega_{b}=-\sum_{c=1}^{m}\left[d f_{i c}+\sum_{j=1}^{m} f_{j c} \phi^{*} \eta_{j i}+\sum_{b=1}^{m} f_{i b} \omega_{b c}\right] \wedge \omega_{c a},
\end{gathered}
$$




$$
\sum_{b=1}^{m} \sum_{j=1}^{n} f_{j a b} \phi^{*} \eta_{j i} \wedge \omega_{b}=-\sum_{j=1}^{n}\left[d f_{j a}+\sum_{k=1}^{m} f_{k a} \phi^{*} \eta_{k j}+\sum_{b=1}^{m} f_{j b} \omega_{b a}\right] \wedge \phi^{*} \eta_{j i} .
$$

Now Equation 4.1 follows from using these relations and second structure equations in the above equation and from the observation

$$
\begin{gathered}
\sum_{b, c=1}^{m} f_{i c} \omega_{c b} \wedge \omega_{b a}=\sum_{b, c=1}^{m} f_{i b} \omega_{b c} \wedge \omega_{c a}, \\
\sum_{j, k=1}^{n} f_{k a} \phi^{*} \eta_{k j} \wedge \phi^{*} \eta_{j i}=\sum_{j, k=1}^{n} f_{j a} \phi^{*} \eta_{j k} \wedge \phi^{*} \eta_{k i} .
\end{gathered}
$$

To prove Equation 4.3 we use Equations 4.1, 4.2 to have

$$
\sum_{b, c=1}^{m}\left[\left(f_{i a b c}-f_{i a c b}\right) \omega_{c} \wedge \omega_{b}\right]=\sum_{b=1}^{m} f_{i b} \Omega_{b a}^{M}+\sum_{c=1}^{m} f_{i c} \Omega_{c a}^{M}+2 \sum_{j=1}^{n} f_{j a} \phi^{*} \Omega_{j i}^{N} .
$$

The result now follows directly from the definitions of the pull back map and curvature forms.

With the above Lemma on hand, we proceed to compute the Laplacian.

Proposition 4.2. Let $\phi:\left(M^{m}, g\right) \rightarrow\left(N^{n}, h\right)$ be a horizontally (weakly) conformal map with dilation $\lambda$. Then at $x \in M$,

$$
-\frac{n}{2} \Delta \lambda^{2}=\sum_{i=1}^{n} \sum_{a, c=1}^{m}\left[\left(f_{i a c}\right)^{2}+f_{i a} f_{i c c a}\right]+\lambda^{2} \sum_{a=1}^{n} \mathbf{R}_{a a}^{M}-\lambda^{4} \mathbf{S c a l}^{\mathbf{N}} .
$$

Proof. Recall from Lemma 2.1 that $\Delta \lambda^{2}$ satisfies

$$
-\frac{n}{2} \Delta \lambda^{2}=\frac{n}{2} \sum_{c=1}^{m} \lambda_{c c}^{2}
$$

where the functions $\lambda_{c b}^{2}$ are determined by

$$
d\left(\lambda_{c}^{2}\right)-\sum_{b=1}^{m} \lambda_{b}^{2} \omega_{c b}=\sum_{b=1}^{m} \lambda_{c b}^{2} \omega_{b}
$$

But from Lemma 4.1, the functions $\lambda_{b}^{2}$ are

$$
\lambda_{b}^{2}=\frac{2}{n} \sum_{i=1}^{n} \sum_{a=1}^{m} f_{i a} f_{i a b} .
$$

Hence

$$
d\left(\lambda_{c}^{2}\right)=\frac{2}{n} \sum_{i=1}^{n} \sum_{a=1}^{m}\left[d f_{i a} f_{i a c}+f_{i a} d f_{i a c}\right] .
$$

Using Equations 3.1 and 4.2 for $d f_{i a}, d f_{i a c}$ respectively, we can write

$$
d\left(\lambda_{c}^{2}\right)=\frac{2}{n} \sum_{i=1}^{n} \sum_{a, b=1}^{m}\left[\left\{f_{i a c} f_{i a b}+f_{i a} f_{i a c b}\right\} \omega_{b}-f_{i a} f_{i a b} \omega_{b c}\right] .
$$


Substituting Equation 4.6 and the above equation in Equation 4.5 determines functions $\lambda_{c b}^{2}$ as

$$
\lambda_{c b}^{2}=\frac{2}{n} \sum_{i=1}^{n} \sum_{a=1}^{m}\left[f_{i a c} f_{i a b}+f_{i a} f_{i a c b}\right] .
$$

Hence from Equation 4.4

$$
-\frac{n}{2} \Delta \lambda^{2}=\sum_{i=1}^{n} \sum_{a, c=1}^{m}\left[\left(f_{i a c}\right)^{2}+f_{i a} f_{i a c c}\right] .
$$

Since the functions $f_{i a b c}$ satisfy $f_{i a b c}=f_{i b a c}$ we have

$$
f_{i a c c}=f_{i c a c}=f_{i c c a}-\sum_{d=1}^{m} f_{i d} \mathbf{R}_{d c a c}^{M}-\sum_{j, k, l=1}^{n} f_{j c} f_{k c} f_{l a} \mathbf{R}_{j i k l}^{N},
$$

where we have used Equation 4.3 for the second equality. Thus we have the final expression for the Laplacian as

$$
\begin{array}{r}
-\frac{n}{2} \Delta \lambda^{2}=\sum_{i=1}^{n} \sum_{a=1}^{m}\left[\sum_{c=1}^{m}\left\{\left(f_{i a c}\right)^{2}+f_{i a} f_{i c c a}+\sum_{j, k, l=1}^{n} f_{i a} f_{j c} f_{k c} f_{l a} \mathbf{R}_{j i k l}^{N}\right\}\right. \\
\left.+\sum_{d=1}^{m} f_{i a} f_{i d} \mathbf{R}_{d a}^{M}\right] .
\end{array}
$$

But we know from Remark 3.5 that (locally) we can choose a $\phi$-adapted framing for a horizontally (weakly) conformal map such that $f_{i a}=\lambda \delta_{i a}$. The required expression follows by writing $-\Delta \lambda^{2}$ for a $\phi$-adapted framing.

From Equation 4.2 and the skewness of $\omega_{a c}$, we notice that $\sum_{c, a} f_{i c c a} \omega_{a}=0$ if $\phi$ is harmonic. Hence the characterization of harmonic morphisms as harmonic horizontally (weakly) conformal maps leads to the following Weitzenböck formula for harmonic morphisms.

Corollary 4.3. Let $\phi:\left(M^{m}, g\right) \rightarrow\left(N^{n}, h\right)$ be a harmonic morphism between Riemannian manifolds. Then the dilation $\lambda$ of $\phi$ satisfies

$$
-\frac{n}{2} \Delta \lambda^{2}=\sum_{i=1}^{n} \sum_{a, c=1}^{m}\left(f_{i a c}\right)^{2}+\left.\lambda^{2} \mathbf{S} \mathbf{c a l}{ }^{\mathbf{M}}\right|_{\mathbf{H}}-\lambda^{4} \mathbf{S c a l}^{\mathbf{N}},
$$

where

$$
\left.\mathbf{S c a l}^{\mathbf{M}}\right|_{\mathbf{H}}=\sum_{a=1}^{n} \mathbf{R}_{a a}^{M} .
$$

This Weitzenböck formula was also obtained in [17] but by a different method.

\section{RESTRICTIONS ON MAPS FROM NON-POSITIVELY CURVED MANIFOLDS}

A standard Bochner type argument applied to the Weitzenböck formula in Corollary 4.3 led to the following restrictions on harmonic morphisms from positively curved compact manifolds, as shown in [17].

Proposition 5.1 ([17]). Let $\phi: M \rightarrow N$ be a harmonic morphism between compact Riemannian manifolds with dilation $\lambda$. 
1. $\phi$ is totally geodesic if $\left.\mathbf{S} \mathbf{c a l}^{\mathbf{M}}\right|_{\mathbf{H}} \geq 0$ and $\mathbf{S c a l}^{\mathbf{N}} \leq 0$. If at some point, either $\left.\mathbf{S c a l}^{\mathbf{M}}\right|_{\mathbf{H}}>0$ or $\mathbf{S c a l}^{\mathbf{N}}<0$, then $\phi$ is constant.

2. Suppose that $\phi$ is submersive and $\left.\mathbf{S} \mathbf{c a l}{ }^{\mathbf{M}}\right|_{\mathbf{H}} \geq A, 0<\mathbf{S c a l}^{\mathbf{N}} \leq B$ for $A, B>$ 0. If $\lambda^{2}$ is bounded by

$$
\lambda^{2} \leq \frac{A}{B} \leq \frac{\left.\mathbf{S c a l}^{\mathrm{M}}\right|_{\mathbf{H}}}{\mathbf{S c a l}^{\mathbf{N}}}
$$

then $\phi$ is totally geodesic.

We refer the reader to $[17, \S 3]$ for a number of examples which satisfy the above hypothesis.

The standard Bochner type argument, though very powerful, is not quite useful in analysing maps from non-positively curved manifolds. To overcome this constraint we develop a scheme based on the following.

Lemma 5.2. Let $\phi:\left(M^{m}, g\right) \rightarrow\left(N^{n}, h\right)$ be a non-constant harmonic morphism with dilation $\lambda$. If

(i) $\lambda^{2}$ attains its maximum at $x \in M$,

(ii) $\mathbf{R i c c i}_{x}^{M} \geq-A \quad(A>0)$,

(iii) $\mathbf{S c a l}_{x}^{N} \leq-B \quad(B>0)$,

then

$$
\lambda^{2} \leq n \frac{A}{B}
$$

In particular, if $B>n A$, then $\phi$ cannot be a Riemannian submersion.

Proof. At the point $x$ we have $-\Delta \lambda^{2} \leq 0$, therefore Corollary 4.3 implies that

$$
\left.\lambda^{2} \mathbf{S c a l}^{\mathbf{M}}\right|_{\mathbf{H}}-\lambda^{4} \mathbf{S c a l}^{\mathbf{N}} \leq-\sum_{i=1}^{n} \sum_{a, c=1}^{m}\left(f_{i a c}\right)^{2} \leq 0 .
$$

Combining this inequality with the hypothesis completes the proof.

Similarly we can show:

Proposition 5.3. Let $\phi: M^{m} \rightarrow N^{n}$ be a non-constant harmonic morphism such that $M$ is Ricci-flat and $\mathbf{S c a l}^{N} \leq-B,(B>0)$. Then $\lambda^{2}$ does not attain its maximum in $M$.

For a compact domain we obtain

Theorem 5.4. Let $M$ be a Ricci flat compact Riemannian manifold and $N$ a Riemannian manifold of constant non-positive scalar curvature. Then a non-constant harmonic morphism $\phi: M \rightarrow N$ can exist only if $N$ has locally zero scalar curvature.

Proof. By compactness of $M$, there exists a point $x \in M$ such that $-\Delta \lambda^{2} \leq 0$. The proof then follows by an argument similar to the proof of Lemma 5.2.

A key step in obtaining further applications of Lemma 5.2 is to ensure that Condition (i) of the hypothesis is satisfied. For suitable spaces, for instance $\mathbb{H}^{m}, \mathbb{R}^{m}$, this can be avoided by adapting a technique of $[9, \S 5]$ to our situation. The main idea is to exhaust $\left(M^{m}, g\right)=\mathbb{H}^{m}, \mathbb{R}^{m}$ by concentric balls $\mathbb{B}_{\alpha}$ and then consider a suitable conformally deformed metric $\tilde{g}=\rho^{2} g$ on $\mathbb{B}_{\alpha}$, such that the restrictions on $\lambda^{2}$ can be obtained from the restrictions on the associated function $\tilde{\lambda}^{2}=\rho^{-2} g$. The 
restrictions on $\tilde{\lambda}^{2}$ are achieved by investigating the Laplacian of $\tilde{\lambda}^{2}$ with respect to $\tilde{g}$ and by showing that $\tilde{\lambda}^{2}$ attains its maximum in $B_{\alpha}$. Precisely we have

Proposition 5.5 (Hyperbolic case). Let $\phi:\left(\mathbb{B}^{m}, g\right) \rightarrow\left(N^{n}, h\right)$ be a non-constant harmonic morphism from a unit open ball such that $g=\left(4 / A\left(1-r^{2}\right)^{2}\right) \sum_{a} d x_{a}^{2}$, with $r^{2}=\sum_{a} x_{a}^{2}$, is a metric of constant negative sectional curvature $-A(A>0)$. Suppose that $\mathbf{S} \mathbf{c a l}^{\mathbf{N}} \leq-B(A>0)$. Then the dilation $\lambda$ of $\phi$ is restricted as

$$
\lambda^{2} \leq n(m-1) \frac{A}{B} .
$$

Proof. Consider an open ball $\mathbb{B}_{\alpha} \subset \mathbb{B}^{m}$ of radius $\alpha<1$, with the conformally deformed metric

$$
\tilde{g}=\frac{4 \alpha^{2}}{A\left(\alpha^{2}-r^{2}\right)^{2}} \sum_{a=1}^{m} d x_{a}^{2}=\left(\frac{\alpha\left(1-r^{2}\right)}{\alpha^{2}-r^{2}}\right)^{2} g=\rho^{2} g
$$

of constant negative sectional curvature $-A$.

First notice that $\phi:\left(\mathbb{B}_{\alpha}, \tilde{g}\right) \rightarrow\left(N^{n}, h\right)$ is not necessarily a harmonic morphism, since $\rho$ is not a constant function. However on $\mathbb{B}_{\alpha}$, we can associate a function $\tilde{\lambda}$ with the dilation $\lambda$ as

$$
\tilde{\lambda}^{2}=\frac{\left\|\phi_{*}\right\|_{\tilde{g}}^{2}}{n}=\rho^{-2} \lambda^{2}=\frac{\left(\alpha^{2}-r^{2}\right)^{2}}{\alpha^{2}\left(1-r^{2}\right)^{2}} \lambda^{2} .
$$

Clearly, the associated function $\tilde{\lambda}^{2}$ attains its maximum on the compact set $\overline{\mathbb{B}_{\alpha}}$, but vanishes on the boundary. Hence $\tilde{\lambda}^{2}$ attains its maximum at $x \in \mathbb{B}_{\alpha}$.

A notational comment: The symbols, (used in previous sections), covered with a tilde shall be considered with respect to the metric $\tilde{g}$, e.g. $\tilde{\omega}_{a}=\rho \omega_{a}$ denote the connection forms with respect to $\tilde{g}$.

If the functions $\tilde{f_{i a}}$ are defined by $\phi^{*} \eta_{i}=\sum_{a} \tilde{f_{i a}} \tilde{\omega}_{a}$, then $\tilde{f_{i a}}=(\lambda / \rho) \delta_{i a}$ with respect to a $\phi$-adapted coframing. Then as in Proposition 4.2 , for a $\phi$-adapted coframing, the Laplacian $-\tilde{\Delta} \tilde{\lambda}^{2}$ can be computed as

$$
-\frac{n}{2} \tilde{\Delta} \tilde{\lambda}^{2}=\sum_{i=1}^{n} \sum_{a, c=1}^{m}\left[\left(\widetilde{f_{i a c}}\right)^{2}+\tilde{f_{i a}} \widetilde{f_{i c c a}}\right]+\left.\frac{\lambda^{2}}{\rho^{2}} \widetilde{\mathbf{S c a l}^{\mathbf{M}}}\right|_{\mathbf{H}}-\frac{\lambda^{4}}{\rho^{4}} \mathbf{S c a l}^{\mathbf{N}} .
$$

From [9, Theorem 5.1] we see that if $\phi$ is a harmonic map with respect to $g$, then $\sum_{i, a, c} \tilde{f_{i a}} \widetilde{f_{i c c a}} \geq 0$. Hence, we can apply the method used in the proof of Lemma 5.2 (above) to have

$$
\begin{aligned}
& \left.\frac{\lambda^{2}}{\rho^{2}} \widetilde{\mathbf{S c a l}^{\mathbf{M}}}\right|_{\mathbf{H}}-\frac{\lambda^{4}}{\rho^{4}} \mathbf{S c a l}^{\mathbf{N}} \leq 0 \\
\text { or }\left.\quad \tilde{\lambda}^{2} \mathbf{S c a l}^{\mathbf{M}}\right|_{\mathbf{H}} & -\tilde{\lambda}^{4} \mathbf{S c a l}^{\mathbf{N}} \leq 0 .
\end{aligned}
$$

Now it is straight forward to show from this inequality that for all $x \in \mathbb{B}_{\alpha}$

$$
\tilde{\lambda}^{2} \leq n(m-1) \frac{A}{B} \text {. }
$$

As Equation 5.2 is true for every open ball $\mathbb{B}_{\alpha}$ of radius $\alpha$ and from Equation 5.1 $\lim _{\alpha \rightarrow 1} \tilde{\lambda}^{2}=\lambda^{2}$ we have $\lambda^{2} \leq n(m-1) \frac{A}{B}$.

Say that the scalar curvature of a manifold $\mathrm{N}$ is negative and bounded away from zero if there exists some $\epsilon>0$ such that $\mathbf{S c a l}^{\mathbf{N}} \leq-\epsilon$. Then for harmonic morphisms from the Euclidean space we have 
Theorem 5.6 (Euclidean case). Consider $\mathbb{R}^{m}$ with the standard metric $g$ of zero curvature. Then there exist no non-constant harmonic morphisms $\phi:\left(\mathbb{R}^{m}, g\right) \rightarrow$ $\left(N^{n}, h\right)$ such that $N$ has negative scalar curvature bounded away from zero.

Proof. Suppose there exists a non-constant harmonic morphism $\phi$ with dilation $\lambda$. As before, consider an open ball $\mathbb{B}_{\alpha} \subset \mathbb{R}^{m}$ of radius $\alpha$, with the conformally deformed metric $\tilde{g}=\left(\alpha^{2} /\left(\alpha^{2}-r^{2}\right)\right)^{2} g=\rho^{2} g$ of negative sectional curvature $-4 / \alpha^{2}$. The associated function $\tilde{\lambda}$ is given by

$$
\tilde{\lambda}^{2}=\frac{\left\|\phi_{*}\right\|_{\tilde{g}}^{2}}{n}=\rho^{-2} \lambda^{2}=\left(\frac{\alpha^{2}-r^{2}}{\alpha^{2}}\right)^{2} \lambda^{2} .
$$

Then following a treatment similar to the above Proposition, along with an argument used in the proof of $[9$, Theorem 5.2], i.e. harmonicity of $\phi$ implies $\sum_{i, a, c} \tilde{f_{i a}} \widetilde{f_{i c c a}} \geq 0$, we have

$$
\tilde{\lambda}^{2} \leq \frac{n(m-1) 4}{\epsilon \alpha^{2}} \quad \forall x \in \mathbb{B}_{\alpha} .
$$

Since Equation 5.4 holds for every $\mathbb{B}_{\alpha}$ of radius $\alpha$ and from Equation $5.3 \lim _{\alpha \rightarrow \infty} \tilde{\lambda}^{2}=$ $\lambda^{2}$ we have $\lambda^{2}=0$, i.e. $\phi$ is constant; a contradiction.

It is known that every compact Riemannian manifold $N^{n}(n \geq 3)$ admits a metric of constant negative scalar curvature (see [15]).

Corollary 5.7. Let $\mathbb{R}^{m}$ be the Euclidean space with standard metric and $N^{n}$ $(n \geq 3)$ be a compact Riemannian manifold. Then there exists a metric $h$ on $N$ such that there are no non-constant harmonic morphisms $\phi: \mathbb{R}^{m} \rightarrow N$ with respect to $h$.

Remark 5.8. Given Riemannian manifolds $(\hat{M}, \hat{g}),(M, g)$ and $(N, h)$, with a smooth map $\phi: M \rightarrow N$ and a harmonic morphism $\pi: \hat{M} \rightarrow M$. Then [11, Proposition 2.1] says that the composition $\hat{\phi}=\phi \circ \pi$ is a harmonic morphism if and only if $\phi$ is a harmonic morphism. Theorem 5.6 combined with this Proposition gives non-existence results for non-constant harmonic morphisms from a non-simplyconnected flat space form to a Riemannian manifold of negative scalar curvature bounded away from zero.

\section{RESTRICTIONS ON HARMONIC MORPHISMS BETWEEN SIMPLY CONNECTED SPACE FORMS}

The characterization of harmonic morphisms implies that harmonic morphisms are solutions of an overdetermined system of partial differential equations. This makes the classification study of harmonic morphisms difficult, even in simpler cases. In case of space forms, a complete classification of harmonic morphisms from 3-dimensional simply connected space forms to 2-dimensional simply connected space forms has been found by Baird-Wood in [2]. For particular cases, some classification results are known in $[3,7,10,14,18,19]$.

Here we present a table which can be utilized in eliminating the possible area of classification of global harmonic morphisms between higher dimensional simply connected space forms. 
Theorem 6.1. Let $\mathbb{E}^{m}$ denote an $m$-dimensional simply connected space form with standard metric of constant sectional curvature 0,1 or -1 . Then we have the following restrictions on global harmonic morphisms $\phi: \mathbb{E}^{m} \rightarrow \mathbb{E}^{n}(m \geq n>1)$ with dilation $\lambda$.

\begin{tabular}{|l|l|l|l|}
\hline $\mathbb{E}^{m}$ & $\mathbb{E}^{n}$ & Type & Restrictions \\
\hline \hline \multirow{4}{*}{$\mathbb{R}^{m}$} & $\mathbb{R}^{n}$ & Any & $\lambda^{2}$ is a subharmonic function. \\
\cline { 2 - 5 } & $\mathbb{S}^{n}$ & Any & $\begin{array}{l}{[10], \phi \text { cannot have totally geodesic fibres and integrable }} \\
\text { horizontal distribution. }\end{array}$ \\
\cline { 2 - 5 } & $\mathbb{H}^{n}$ & Any & $\phi$ is constant. \\
\hline \multirow{4}{*}{$\mathbb{S}^{m}$} & $\mathbb{R}^{n}$ & Any & {$[8], \phi$ is constant. } \\
\cline { 2 - 5 } & $\mathbb{S}^{n}$ & Submersive & If $\phi$ is non-constant, then $\lambda^{2}>(m-1) /(n-1)$. \\
\cline { 2 - 5 } & $\mathbb{H}^{n}$ & Any & {$[8], \phi$ is constant. } \\
\hline \multirow{3}{*}{$\mathbb{H}^{m}$} & $\mathbb{R}^{n}$ & Any & If $\lambda \neq 0$, then $\phi$ cannot be totally geodesic. \\
\cline { 2 - 5 } & $\mathbb{S}^{n}$ & Any & $\begin{array}{l}{[10], \phi \text { cannot have totally geodesic fibres and integrable }} \\
\text { horizontal distribution. }\end{array}$ \\
\cline { 2 - 5 } & $\mathbb{H}^{n}$ & Any & $\lambda^{2} \leq(m-1) /(n-1)$. \\
\hline
\end{tabular}

Proof. The cases $\left(\mathbb{E}^{m}, \mathbb{E}^{n}\right) \in\left\{\left(\mathbb{R}^{m}, \mathbb{R}^{n}\right),\left(\mathbb{H}^{m}, \mathbb{R}^{n}\right)\right\}$ follow from Corollary 4.3. The cases $\left(\mathbb{E}^{m}, \mathbb{E}^{n}\right)=\left(\mathbb{R}^{m}, \mathbb{H}^{n}\right)$ or $\left(\mathbb{H}^{m}, \mathbb{H}^{n}\right)$ are due to Theorem 5.6, Proposition 5.5. For $\left(\mathbb{E}^{m}, \mathbb{E}^{n}\right)=\left(\mathbb{S}^{m}, \mathbb{S}^{n}\right)$ we see that if $\lambda^{2} \leq(m-1) /(n-1)$, then from Proposition $5.1(2) \phi$ is totally geodesic and hence must have constant dilation, totally geodesic fibres and integrable horizontal distribution; a contradiction from the classification of harmonic morphisms between spheres having constant dilation and totally geodesic fibres.

Acknowledgments. The author is very grateful to S. Gudmundsson and J. C. Wood for their useful suggestions. Thanks are also due to the director of the I.C.T.P. for the hospitality and support.

\section{REFERENCES}

[1] Baird, P.: Harmonic maps with symmetry, harmonic morphisms, and deformation of metrics, Pitman Research Notes in Mathematics Series 87, Pitman, Boston, London, Melbourne, 1983. MR 85i:58038

[2] Baird, P. and Wood, J. C.: Harmonic morphisms and conformal foliations by geodesics of three-dimensional space forms, J. Austral. Math. Soc. Ser. A 51 (1991) 118-153. MR 92k:53048

[3] Bryant, R. L.: Harmonic morphisms with fibres of dimension one, Communications in Analysis and Geometry (to appear).

[4] Eells, J. and Lemaire, L.: A report on harmonic maps, Bull. London Math. Soc. 10 (1978) 1-68. MR 82b:58033

[5] Eells, J. and Lemaire, L.: Another report on harmonic maps, Bull. London Math. Soc. 20 (1988) 385-524. MR 89i:58027

[6] Eells, J. and Sampson, J. H.: Harmonic mappings of Riemannian manifolds, Amer. J. Math. 86 (1964) 109-160. MR 29:1603

[7] Eells, J. and Yiu, P.: Polynomial harmonic morphisms between Euclidean spheres, Proc. Amer. Math. Soc. 123 (1995) 2921-2925. MR 95k:58048

[8] Fuglede, B.: Harmonic morphisms between Riemannian manifolds, Ann. Inst. Fourier (Grenoble) 28 (1978) 107-144. MR 80h:58023 
[9] Goldberg, S. I., Ishihara, T. and Petridis N. C.: Mappings of bounded dilatation of Riemannian manifolds, J. Diff. Geometry 10 (1975) 619-630. MR 52:11787

[10] Gudmundsson, S.: Harmonic morphisms between spaces of constant curvature, Proc. Edinburgh Math. Soc. 36 (1993) 133-143. MR 93j:58034

[11] Gudmundsson, S.: Harmonic morphisms from complex projective spaces, Geom. Dedicata $\mathbf{5 3}$ (1994) 155-161. MR 95j:58034

[12] Gudmundsson, S.: The Bibliography of Harmonic Morphisms, http://www.maths.lth.se/matematiklu/personal/sigma/harmonic/bibliography.html.

[13] Ishihara, T.: A mapping of Riemannian manifolds which preserves harmonic functions, $J$. Math. Kyoto Univ. 19 (1979) 215-229. MR 80k:58045

[14] Kasue, A. and Washio, T.: Growth of equivariant harmonic maps and harmonic morphisms, Osaka J. Math. 29 (1992) 899-928. MR 92d:58043; MR 93e:58038

[15] Lohkamp, J.: Metrics of negative Ricci curvature, Ann. of Math. 140 (1994) 655-683. MR 95i: 53042

[16] Montaldo, S.: Harmonic maps and morphisms via moving frames, Lecture notes, University of Leeds (1997).

[17] Mustafa, M. T.: A Bochner technique for harmonic morphisms, J. London Math. Soc. (2) 57 (1998) 746-756. CMP 99:05

[18] Mustafa, M. T. and Wood, J. C.: Harmonic morphisms from three-dimensional Euclidean and spherical space forms, Algebras, Groups and Geometries 15 (1998) 155-172. CMP 99:09

[19] Ou, Y. L. and Wood, J. C.: On the classification of quadratic harmonic morphisms between Euclidean spaces, Algebras, Groups and Geometries 13 (1996) 41-53. MR 97d:58063

[20] Willmore, T. J.: Riemannian geometry, Oxford University Press (1993). MR 95e:53002

[21] Wood, J. C.: Harmonic morphisms, foliations and Gauss maps, Complex differential geometry and nonlinear partial differential equations (Providence, R.I.) (Y.T. Siu, ed.), Contemp. Math. 49, Amer. Math. Soc., Providence, R.I., 1986, 145-184. MR 87i:58045

[22] Wood, J. C.: Harmonic maps and morphisms in 4 dimensions, Geometry, Topology and Physics, Proceedings of the First Brazil-USA Workshop, Campinas, Brazil, June 30-July 7, 1996, B. N. Apanasov, S. B. Bradlow, K. K. Uhlenbeck (Editors), Walter de Gruyter \& Co., Berlin, New York (1997), 317-333. MR 99b:58067

Assistant Professor, Faculty of Engineering Sciences, GiK Institute of Engineering Sciences and Technology, Topi, Distt. Swabi, N.W.F.P., Pakistan

E-mail address: mustafa@giki.edu.pk 